\author{
Military Technical College \\ Kobry El-Kobbah, \\ Cairo, Egypt.
}

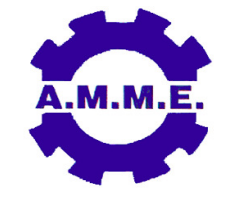

\title{
EVALUATION OF SHAPED CHARGE PERFORMANCE UNDER ITS VARIABLE FACTORS
}

\author{
S. A. Ahmed*, A. M. Riad** and A. Z. Ibrahim**
}

\begin{abstract}
In this paper, two main research directions are used to investigate the shaped charge phenomenon and to evaluate its performance. These directions are: (i) analytical and (ii) numerical simulation. The analytical direction is presented by a model consisting of three main phases; these are: (i) jet formation, (ii) jet break-up, and (iii) jet penetration phases. The governing equations that predict the main parameters associated with each phase are presented. These equations are compiled into a computer program; the input data to the model is that of shaped charge model xx. In numerical simulation direction, Autodyn-2D hydrocode is used to simulate the $\mathrm{xx}$ shaped charge phenomena and to assess the predictions of the analytical model.
\end{abstract}

The predictions of the analytical model are compared with the corresponding measurements of the tested shaped charges of Ref. [8]; good agreement is generally obtained. In addition, the present results of both research directions are mainly concerned with predicting the effects of some factors of shaped charge model $x x$ on its performance. These factors are: (i) liner thickness, (ii) liner material, (iii) cone angle, (iv) type of explosive and (v) standoff distance. Samples of the predicted results representing the effect of each studied factor on the associated parameters with jet formation and jet penetration phases are presented with relevant analysis and discussions. It was found that the analytical results and the corresponding code predictions were consistent. The obtained results recommended the use of powerful explosive, the decrease of cone angle and the increase of standoff distance, keeping the jet continuity, for increasing the performance of shaped charge model $x \mathrm{x}$.

\section{KEY WORDS}

Shaped charges, jet formation, Modified Bernoulli's equation, shaped charge performance, Autodyn-2D, modeling and simulation.

* $\quad$ Military Factory No. 99, National Authority for Military Production.

** Egyptian Armed Forces. 


\section{INTRODUCTION}

The importance of civil and military applications of shaped charges pays the attention of many investigators to extensively study the shaped charge phenomena. Great efforts have been achieved to improve the shaped charge performance. Many investigators studied the effect of numerous factors of shaped charge on its performance. The development of computer codes and the advanced techniques for experimentation enriche this field. However, the use of analytical models is still regarded as the fast and cheap tool for fulfilling the deisgn and optimization requirements put on shaped charges.

Chanteret and Lichtenberger [1] studied experimentally the effect of liner wall thickness on jet formation process. They found that the increase of liner thickness decreased the jet velocity and increased the velocity difference between successive break-up jet elements. Mayseless at al. [2] investigated the effect of liner thickness on jet formation by comparing their experimental results with that predicted analytically and numerically. Their findings were: (i) liner of thin wall produced a long jet with thick tip, and (ii) the maximum jet penetration into a hard target at a long standoff distance was obtained when the liner thickness was less than $2 \%$ of the charge diameter.

Chanteret [3] investigated numerically the influence of liner density on jet tip velocity and shaped charge performance. He fed the ISLID code with data of $50^{\circ}$ conical liners with a 95\% HMX content explosive loading. The code was run using different thicknesses of liners made of aluminium, copper and tungsten, respectively. $\mathrm{He}$ found that: (i) the total length of jet decreased with the liner density to the power $1 / 2$ or $1 / 3$ depending on the used break-up model, and (ii) the maximum performance of particulated jet was independent of liner density.

Saran et al. [4] investigated experimentally the performance of aluminium lined shaped charges. Their tested liners having different thicknesses $(4 \%, 6 \%$ and $8 \%$ of charge diameter (CD)) and geometries (conical and trumpet) with different alloy compositions (5000, 6000, and 7000-series aluminium alloys) were fired against steel targets at standoff of $3 \mathrm{CD}$ and $15 \mathrm{CD}$, respectively. They found that: (i) aluminium alloy of series 6000 gave the maximum penetration depth compared with that of other tested aluminium alloys, (ii) the penetration of the trumpet liners was more deeper into the steel target, and (iii) the decrease in liner thickness had an increasing effect on penetration depth with minor decrease in hole diameter.

Baker et al. [5] investigated numerically and experimentally the influence of a powerful explosive formulation on the shaped charge performance. They used the JAGUAR computer program for determining the parameters of Jones-Wilkins-LeeBaker (JWLB) equation of state. Then, they fed the CALE computer program with the data of shaped charge having a diameter of $81.3 \mathrm{~mm}$, a constant liner thickness, and a cone angle of $42^{\circ}$. The program was fed with the data of powerful explosive formulation and $L X-14$, respectively. They performed an experimental program by shaped charges loaded with PAX-16 formulation and LX-14, respectively. They found the followings: (i) the loading of explosive formulation in shaped charge produced a longer jet length than that loaded by LX-14, with an associated increase 
in break-up time and penetration depth at large standoff, and (ii) the energy resulted from the formulation had great effect on increasing the shaped charge performance.

Pertanian and Prelhad [6] developed a model analysing the temperature distribution during jet formation process. They assumed that the formed jet was melted under tremendous temperature and pressure, and flew like a liquid. They fed the model with the data of different explosive materials (RDX, TNT and Nitro-glycerine (NG)) and different liner materials (steel, lead and copper). They found the followings: (i) the temperature increased with axial distance, and (ii) maximum temperature was attained by RDX in comparison with other explosive materials. They also reported that their findings would help in determining the type of liner which can be used in shaped charge.

Chuang et al. [7] investigated experimentally the jet penetration formed from a biconical liner shaped charges into steel targets at different standoff distances. They obtained the optimum standoff of biconical liner with the change of top cone angle and they established an empirical equation correlating the penetration depth with top cone angle and standoff distance using interpolation method. Their empirical equation showed that the maximum penetration depth decreased with increasing the top cone angle. They also predicted that the optimum standoff distance increased with the increase of top cone angle and bottom cone angle using Autodyn-2D hydrocode. In addition, they found, according to their tests, simulation and the established empirical equation, that the maximum jet penetration into steel targets can be obtained by the combination of different top and bottom cone angles.

In this paper, a proposed analytical model is used to describe the shaped charge phenomenon and to predict the influence of each shaped charge factor on its performance. The present model consists of three main phases; these are: (i) jet formation, (ii) jet break-up, and (iii) jet penetration phases. The govening equations of each phase are presented, arranged and compiled into a computer program. In addition, Autodyn-2D hydrocode is used to simulate the shaped chrage jet formation and jet penetration into Rolled Homogenous Armor (RHA). Both the analytical model and the Autodyn-2D hydrocode are fed with the data of shaped charge model $x x$. The effect of each factor of the shaped charge model $x x$ on its performance is evaluated by comparing the depths of jet penetration and the crater radii at the target surface with that of the original shaped charge. Representative samples of the obtained results analytically and numerically are presented and discussed.

\section{ANALYTICAL MODEL}

In the following, the one-dimensional analytical model of Mohamed [8] is chosen for the present study. This model has been modified by: (i) replacing the formulae that predicts the initial length of jet and the jet length prior to impact (effective length) into a target by new ones, (b) adding the influence of standoff distance on the effective length of the formed jet, and (iii) considering the compression in jet length during its penetration through the target.

The proposed model is divided into three main phases; these are: a) jet formation, b) jet break-up, and c) jet penetration into a metallic target. For each phase, the shaped 
charge liner or the formed jet is divided into $\mathrm{n}$ elements. Figure 1 presents a schematic drawing of a collapsed liner element during jet formation phase [9]. In the following, the governing equations representing each phase of the analytical model are presented. For jet formation phase, the governing equations are that predict the deflection angle, collapse angle, real collapse velocity, jet velocity and jet mass associated with each collapsed element of liner. For jet break-up phase, a semiempirical formula, developed by Hennequin [10], is selected to predict the break-up time of each jet element.

A schematic drawing representing the jet penetration into a target can be shown in Fig. 2 [9]. The governing equations of jet penetration phase, that predict the penetration velocity, penetration depth and radius of crater at the target surface associated with each penetrated element of jet, are also presented. The total depth of penetration for a particualted jet can be predicted by the present model.

In the following, the governing equations representing each phase of the proposed analytical model are presented. The interested reader could be referred to the original reference for further details [11].

\section{Governing Equations of Jet Formation Phase}

a) The real collapse velocity, $V_{\text {real }}$, of the collapsed liner element at the charge axis is represented by [9]:

$$
V_{\text {real }}=V_{0}\left(1-e^{-\frac{t_{t}-t_{0}}{T}}\right)
$$

where $V_{o}$ is the collapse velocity of the liner element, $t_{f}$ is the time at which the collapsed liner element reaches the charge axis, $t_{0}$ is the time taken by the detonation wave front to reach the liner element, and $\mathrm{T}$ is time constant.

b) The deflection angle $\delta$ of each collapsed liner element is represented by:

$$
\delta=\sin ^{-1}\left(\mathrm{~V}_{\text {real }} \cos \alpha / 2 \mathrm{U}_{\mathrm{D}}\right)
$$

where $\alpha$ is the half of the apex angle.

c) The collapse angle $\beta$ associated with each collapsed liner element is determined by [12]:

and

$$
\beta=\left(\beta^{+}+\Delta \beta\right)
$$

$$
\beta^{+}=\alpha+2 . \delta
$$

where $\beta^{+}$is the collapse angle obtained by steady state theory [9]. The angle $\Delta \beta$ is determined using the following equation [12]:

$$
\Delta \beta=\tan ^{-1}\left\{-\left(\mathrm{X}_{i} \sin \alpha /(\cos (\alpha+\delta) \cdot \cos \delta)\right) .\left(\mathrm{V}_{\text {real }}^{\prime} / \mathrm{V}_{\text {real }}\right)\right\},
$$

where $V_{\text {real }}^{\prime}$ is the partial derivative of the real collapse velocity with respect to the distance of the liner element from the liner apex; this partial derivative is determined using the following equation: 


$$
V_{\text {real }}^{\prime}=\left(d V_{\text {real }} / d X_{i}\right)=\left(d V_{0} / d \mu\right) \cdot\left(d \mu / d X_{i}\right) \cdot\left(1-e^{-\frac{t_{f}-t_{0}}{T}}\right)
$$

d) The velocity of jet generated from each collapsed element of liner, $V_{j}$, is represented by [9]:

$$
V_{j}=V_{\text {real }} \csc (\beta / 2) \cdot \cos (\alpha+\delta-(\beta / 2))
$$

e) The mass of jet generated from each collapsed element of liner, $\mathrm{m}_{\mathrm{ji}}$, is represented by [9]:

$$
m_{j_{i}}=M_{L_{i}} \sin ^{2}(\beta / 2) \text {. }
$$

where $\mathrm{M}_{\mathrm{Li}}$ is the mass of collapsed element of liner.

\section{Governing Equations of Jet Break-up Phase}

The break-up time of each jet element, $t_{b i}$, is determined using the semi-empirical formula developed by Hennequin [10]. The selected formula in its final form is represented by:

$$
\mathrm{t}_{\mathrm{bi}}=\text { 2.92. }\left(\mathrm{r}_{\mathrm{ji}} / \mathrm{V}_{\mathrm{pl}}\right)+0.46 .\left(\mathrm{L}_{\mathrm{i}} / \mathrm{V}_{\mathrm{pl}}\right)
$$

where $r_{j_{i}}$ is the initial radius of each jet element, $\mathrm{V}_{\mathrm{pl}}$ is the velocity difference between the jet particulated elements, and $L_{i}$ is the length of the particulated jet element. The velocity difference between particualted jet elements is represented by [13]:

$$
\mathrm{V}_{\mathrm{pl}}=\left(\mathrm{V}_{\max }-\mathrm{V}_{\min }\right) / \mathrm{n}_{\mathrm{f}}
$$

where $V_{\max }$ is the velocity of front element of the formed jet, $V_{\min }$ is the velocity of rear element of the formed jet, and $n_{f}$ is the number of elements resulted from the jet break-up.

\section{Governing Equations of Jet Penetration Phase}

a) The initial length of jet, $\mathrm{l}_{\mathrm{j}}$, is represented by [14]:

$$
\mathrm{I}_{j}=\mathrm{K}_{H}(\alpha) \mathrm{I}=\mathrm{K}_{\mathrm{H}}(\alpha) \cdot \frac{\left(\mathrm{R}_{\mathrm{B}}-\frac{\mathrm{t}_{\mathrm{I}}}{\cos \alpha}\right)}{\sin \alpha},
$$

where $I$ is the length of liner wall, $R_{B}$ is the base radius of the conical liner, $t_{1}$ is the thickness of liner wall and $\alpha$ is the half of cone angle. For each liner material, the coefficient $\mathrm{K}_{\mathrm{H}}$ depends on the angle of conical liner.

b) The length of jet prior to impact (effective length), $L_{e}$, is determined by:

$$
I_{e}=I_{j}+I \cos \alpha \cdot\left[1-\frac{I_{m}}{I}\left(1+\frac{\tan \alpha}{\tan \beta_{m}}\right)\right]+\operatorname{SoD}
$$


where $I_{m}$ is the length of collapsed liner at which the jet element moves with maximum jet velocity, $\beta_{m}$ is the corresponding collapse angle with charge axis and SoD is the standoff distance between liner base and target surface.

c) The cumulative length of jet, $L_{c}$, is determined by:

$$
\mathrm{I}_{\mathrm{c}}=\mathrm{I}_{\mathrm{j}}+\left(\mathrm{V}_{\max }-\mathrm{V}_{\min }\right) * \mathrm{t}_{\mathrm{b} 1},
$$

where $t_{b 1}$ is the break-up time of the first element of jet. Two statuses were considered for the formed jet; they are continuous and particulated. The jet status is considered to be continuous if $\mathrm{I}_{\mathrm{e}}<\mathrm{I}_{\mathrm{c}}$, and to be particulated If $\mathrm{I}_{\mathrm{e}}>\mathrm{I}_{\mathrm{C}}$.

d) The proposed formula of Eichelberger that incorporates the strengths of jet and target materials [9]:

$$
\lambda \cdot \rho_{j}\left(V_{i}-U_{i}\right)^{2}=\rho_{t} U_{i}^{2}+2 \sigma
$$

and

$$
\sigma=\sigma_{\mathrm{t}}-\sigma_{\mathrm{j}},
$$

where $\lambda$ is a constant which is equal to one for a continuous jet and less than one for a particulated jet [15], $\rho_{j}$ is the density of jet material, $V_{i}$ is the velocity of jet penetrated element, $U_{i}$ is the penetration velocity, $\rho_{t}$ is the density of target material, $\sigma_{t}$ is the strength factor of target material, and $\sigma_{j}$ is the strength factor of jet material.

e) The penetration velocity for each penetrated jet element, $U_{i}$, is represented by:

$$
\mathrm{U}_{\mathrm{i}}=\frac{\mathrm{V}_{\mathrm{i}}-\sqrt{\varphi \cdot \mathrm{V}_{\mathrm{i}}^{2}+\mathrm{A} \cdot(1-\varphi)}}{(1-\varphi)}
$$

and

$$
\varphi=\frac{\rho_{\mathrm{t}}}{\lambda \cdot \rho_{\mathrm{j}}} \quad \text { and } \quad \mathrm{A}=\frac{2 \sigma}{\lambda \cdot \rho_{\mathrm{j}}} .
$$

f) The decreasing rate of length of jet penetrating element, $L_{\mathrm{j} j}$, with respect to time is:

$$
\frac{d L_{j i}}{d t}=-\left(V_{i}-U_{i}\right) \text {. }
$$

g) The rate of change of penetration depth for each penetrating element, $Z_{i}$, with respect to time is:

$$
\frac{d Z_{i}}{d t}=U_{i}
$$

h) The decrease in jet length due to its compression, $L_{j c}$, during the penetration of the interacting element of jet with the target is represented by:

$$
\mathrm{L}_{\mathrm{jc}}=\left(\left(\mathrm{V}_{\mathrm{i}}-\mathrm{U}_{\mathrm{i}}\right)-\mathrm{V}_{\text {tail }}\right) * \Delta \mathrm{t} \text {. }
$$

i) For a particulated jet, the depth of penetration into a target, $Z$ ', is calculated using a formula developed by Carleone et. al. [See Ref. [16]]: 


$$
Z^{\prime}=Z^{*}\left(1-\frac{g}{g_{0}}\right)
$$

where $\mathbf{Z}$ is the total depth of penetration of continuous jet, $\mathrm{g}$ is the sum of gap distances between the break-up jet elements, and $g_{0}$ is an empirical constant. The total air gaps distance between the particulated elements of jet are considered to be equal to $\left(I_{e}-I_{c}\right)$.

j) The crater radius, $r_{c}$, as a function of time, $t_{C}$, is determined by:

where

$$
r_{c}=\sqrt{\frac{A}{B}-\left(\sqrt{\frac{A}{B}-r_{j i}^{2}}-t_{c} \sqrt{B}\right)^{2}},
$$

$$
A=\frac{2 r_{j i}^{2} p_{o}}{\rho_{t}}, B=2 \frac{\sigma_{t}}{\rho_{t}} \text { and } P_{o}=\frac{1}{2} \rho_{t} U_{i}^{2}+\sigma_{t},
$$

k) The radius of the penetrated jet element $r_{j i}$ is determined by:

$$
r_{j i}=\sqrt{\frac{m_{j i}}{\pi L_{j i} \rho_{j}}},
$$

where $m_{j i}$ is the mass of penetrated jet element, and $L_{j i}$ is the length of the penetrated element of jet.

The governing equations of the proposed model are arranged and compiled into a computer program. The input data to the program are easily determined. In the following, the predicted results of the present model are essentially concerned with studying the effect of each $x x$ shaped charge factor on its performance.

\section{NUMERICAL SIMULATION}

In the following, Autodyn-2D hydrocode is used to investigate the influence of each factor of the shaped charge model $x x$ on the penetration depth of the formed jet into $\mathrm{RHA}$ and the crater radius at the target surface. The studied factors are: (i) liner thickness, (ii) liner material, (iii) cone angle, (iv) type of explosive and (v) standoff distance between the cone base and target surface. Figure 3 shows a diagrammatic scheme of the shaped charge model xx including its main dimensions [17] whereas; the span of each studied factor of this shaped charge can be shown in Table 1.

\section{Model Description}

Autodyn-2D hydrocode is provided with the data of elements of shaped charge model $x x$ to simulate jetting analysis, jet formation and jet penetration into monolithic semi- infinite RHA processes, respectively. In the following, descriptions of shaped charge elements, their materials with their equations of state and strength models, are introduced. Moreover, the main features for simulating the shaped charge jetting analysis, jet formation, and the interaction of the formed jet with monolithic $\mathrm{RHA}$, respectively, are also presented. 
Table 1. Span of the studied factors of shaped charge model xx.

\begin{tabular}{|c|c|c|c|c|}
\hline \multirow{2}{*}{ Factor } & \multicolumn{4}{|c|}{ Shaped charge dimensions } \\
\hline & Lower & & & greater \\
\hline Liner Thickness, $\mathrm{T}_{\mathrm{L}}[\mathrm{mm}]$ & 1.0 & & & 1.4 \\
\hline Liner material & & Copper & Steel & \\
\hline Half of apex angle, $\alpha\left[{ }^{\circ}\right]$ & 25 & & & 35 \\
\hline Type of explosives & TNT & & & HMX \\
\hline Standoff Dist., SoD [mm] & - & & & 26 , and 38 \\
\hline
\end{tabular}

\section{Description of the charge case}

The function of the shaped charge case is to provide confinement for the explosive charge. The equation of state for the used steel material was linear. The present form of this equation considering the initial elastic behavior expressed by an approximation to Hooke's Law can be written as [18]:

$$
\mathrm{P}=\mathrm{K} \cdot \mu
$$

where $P$ is the pressure, $\mu$ is the compressibility and is equal to $\left[\left(\rho / \rho_{0}\right)-1\right], K$ is the bulk modulus of case material, $\rho_{0}$ is the reference density and $\rho$ is the current density [18]. The input data to Autodyn-2D hydrocode for the case material are listed in Table 2.

\section{Description of the liner}

The material used for filling the meshes of liner elements was copper. The equation of state for the used material was shock and the strength model was neglected due to the very high shock pressure compared with the strength of liner material. In the present work, the equation of state for liner material is expressed by [18]:

$$
P=P_{H}+\Gamma \cdot \rho .\left(e-e_{H}\right)
$$

where $\Gamma$ is the Gruneisen gamma coefficient and is equal to $\left[B_{0} /(1+\mu)\right]$; $B_{0}$ is a constant and $\mu$ is the compressibility $\left(=\left(\rho / \rho_{0}\right)-1\right), P_{H}$ and $e_{H}$ are the Hugoniot pressure and energy, respectively, given by:

$$
P_{H}=\frac{\rho_{0} \cdot c_{0}^{2} \cdot \mu \cdot(1+\mu)}{(1-(s-1) \mu)^{2}},
$$

and

$$
\mathrm{e}_{\mathrm{H}}=\frac{1}{2} \cdot \frac{\mathrm{P}_{\mathrm{H}}}{\rho_{\mathrm{o}}} \cdot\left(\frac{\mu}{1+\mu}\right),
$$

where $c_{o}$ is the sound velocity in the material, and $s$ is constant giving the slope of shock velocity-particle velocity relationship. More detailed information about the used equation of state is reported in Ref. [18]. 
Table 2. Input data to the code for the charge case material.

\begin{tabular}{|l|c|}
\hline \multicolumn{1}{|c|}{ Parameter } & Value \\
\hline Reference density, $\left(\mathrm{kg} / \mathrm{m}^{3}\right)$ & 7800 \\
\hline Bulk modulus, $(\mathrm{GPa})$ & 160 \\
\hline Reference temperature, $(\mathrm{K})$ & 300 \\
\hline Shear modulus, $(\mathrm{GPa})$ & 74.5 \\
\hline Yield stress, $(\mathrm{MPa})$ & 400 \\
\hline Hydro tensile limit, $\mathrm{P}_{\min }(\mathrm{MPa})$ & $-2 \times 10^{3}$ \\
\hline
\end{tabular}

Autodyn-2D hydrocode was fed with the data listed in Table 3 for the copper and steel liner materials with wall thickness of $1.2 \mathrm{~mm}$. The constants of the previous equations were taken from the material library of the code. The influence of changing the copper liner wall thicknesses with 1 and $1.4 \mathrm{~mm}$, respectively, on the shaped charge performance are also studied considering the listed data in Table 3.

\section{Description of the explosive}

The materials used for filling the meshes of the explosive inside the shaped charge model xx were TNT, Hexogen and HMX, respectively. The equation of state for the used explosives was "Jones-Wilkins-Lee" (JWL) equation, which is represented by [18]:

$$
P=A \cdot e^{-r_{1} v}+B \cdot e^{-r_{2} v}+C \cdot v^{-(1-\omega)},
$$

where $P$ is the pressure, $v$ is the relative volume, $A, B, r_{1}, r_{2}, C$ and $\omega$ are constants. For each of the used explosives, the values of the previous constants were available in the material library of the used hydrocode. The input data to Autodyn-2D hydrocode for each of the studied explosive materials are listed in Table 4.

\section{Simulation of Shaped Charge Processes}

\section{Jetting analysis process}

It is the simplest process that predicts the parameters associated with jet formation. The liner was described as a thin shell composed of a series of nodes having the real thickness of the liner, while its apex point should be fixed by a boundary condition to prevent its motion. The interaction between the shell grids of liner with the grids for explosive and case is defined by Euler-Lagrange polygon surface. Jetting analysis calculations were based on PER theory of jet formation [9]. Figure 4 illustrates the conical $x x$ shaped charge assembly including the Eulerian case and explosive as well as the shell liner with fixed apex node as a complete representation of jetting analysis process. The main steps used to simulate the jetting analysis process are reported in Ref. [11].

\section{Jet formation process}

The jet formation process can predict the profile of the formed jet as function of time, 
Table 3. Input data to the code for the liner materials.

\begin{tabular}{|l|c|l|c|}
\hline \multicolumn{2}{|c|}{ Copper liner } & \multicolumn{2}{c|}{ Steel liner } \\
\hline \multicolumn{1}{|c|}{ Parameter } & Value & \multicolumn{1}{c|}{ Parameter } & Value \\
\hline Equation of state, & shock & Equation of state, & shock \\
\hline Strength model, & None & Strength model, & None \\
\hline Reference density, $\left(\mathrm{kg} / \mathrm{m}^{3}\right)$ & 8930 & Reference density, $\left(\mathrm{kg} / \mathrm{m}^{3}\right)$ & 7800 \\
\hline Gruneisen coefficient & 2.02 & Gruneisen coefficient & 2.17 \\
\hline Sound speed, $\mathrm{C}_{\mathrm{o}}(\mathrm{m} / \mathrm{s})$ & 3940 & Sound speed, $\mathrm{C}_{\mathrm{o}}(\mathrm{m} / \mathrm{s})$ & 4590 \\
\hline Parameter, S & 1.489 & Parameter, S & 1.49 \\
\hline Reference temp., $(\mathrm{K})$ & 300 & Reference temp., $(\mathrm{K})$ & 300 \\
\hline
\end{tabular}

Table 4. Input data to the code for TNT, RDX and HMX materials, respectively.

\begin{tabular}{|l|c|c|c|}
\hline \multirow{2}{*}{ Parameter } & \multicolumn{3}{|c|}{ Explosive type } \\
\cline { 2 - 4 } & TNT & RDX & HMX \\
\hline Density, $\left(\mathrm{kg} / \mathrm{m}^{3}\right)$ & 1630 & 1770 & 1890 \\
\hline Parameter, $\mathrm{A}(\mathrm{kPa})$ & $3.740 \times 10^{8}$ & $6.539 \times 10^{8}$ & $7.782 \times 10^{8}$ \\
\hline Parameter, B $(\mathrm{kPa})$ & $3.747 \times 10^{6}$ & $7.293 \times 10^{7}$ & $7.071 \times 10^{6}$ \\
\hline Parameter, $\mathrm{r}_{1}$ & 4.15 & 4.83 & 4.2 \\
\hline Parameter, $\mathrm{r}_{2}$ & 0.9 & 2.24 & 1 \\
\hline C-J detonation velocity, $(\mathrm{m} / \mathrm{s})$ & 6930 & 8700 & 9100 \\
\hline $\begin{array}{l}\text { C-J energy/unit volume, } \\
\left(\mathrm{KJ} / \mathrm{m}^{3}\right)\end{array}$ & $6.00 \times 10^{6}$ & $5.62 \times 10^{6}$ & $1.05 \times 10^{7}$ \\
\hline C-J pressure, $(\mathrm{kPa})$ & $2.1 \times 10^{7}$ & $2.6 \times 10^{7}$ & $4.2 \times 10^{7}$ \\
\hline Parameter, $\omega$ & 0.3 & 0.3 & 0.3 \\
\hline
\end{tabular}

the contours of different jet parameters and the jet break-up. In addition, the performance of the formed jet is strongly affected by the initial detonation wave front incident upon the shaped charge liner. The initiation method affects the detonation wave shape and the associated pressure, which are considered as one of the factor influencing the shaped charge performance. The main procedures for simulating the jet formation process and describing the initiation point for the explosive charge are reported in Ref. [11], respectively.

\section{Simulation of jet penetration into RHA}

After terminating the jet formation process, the jet penetration into monolithic semiinfinite RHA was simulated considering each of the studied factors of shaped charge model $x x$. A certain block of RHA having a Brinell hardness number ranged from 280-320 was added to the model. This material was fed to the hydrocode by a linear equation of state. Johnson Cook strength model was also selected. The Johnson Cook equation defines the dynamic yield strength $Y$ as [18]:

$$
\mathrm{Y}=\left(\mathrm{A}+\mathrm{B} \cdot \varepsilon^{\mathrm{n}}\right)\left(1+C \cdot \ln \varepsilon_{P}^{*}\right)\left(1-T_{H}^{m}\right)
$$

where $\varepsilon$ is the effective plastic strain, $A$ is the yield strength, $B$ is the hardening constant, $\mathrm{n}$ is the hardening exponent, $\mathrm{C}$ is the strain rate constant, $\mathrm{m}$ is the thermal exponent constant, $\varepsilon_{P}{ }^{*}$ is the normalized effective plastic strain rate; i.e. the applied 
true strain-rate divided by reference strain-rate), and $T_{H}$ is the homologous temperature.

The expression in the first set of brackets gives the stress as a function of strain when $\varepsilon_{\mathrm{p}}{ }^{*}=1.0 \mathrm{sec}^{-1}$ and $\mathrm{T}_{\mathrm{H}}=0$. The constant $A$ is the basic yield stress at low strains while $B$ and $n$ represent the effect of strain hardening. The expressions in the second and third sets of brackets represent the effects of strain rate and temperature, respectively. Input data of the target material to the hydrocode are listed in Table 5.

The penetration process has been terminated when the nodes' velocities fall below the cutoff velocity. Moreover, pressure contours, eroded jet nodes, stresses, and type of failure could be identified. Finally, both the penetration depth and crater radius at the target surface were predicted.

\section{RESULTS AND DISCUSSIONS}

In the following, the present results are concerned with that of the shaped charge model XX. However, the proposed model and the Autodyn-2D hydrocode could predict many results for each of their phases, the introduced results is concerned with the effect of each shaped charge factor on its performance. Therefore, The present results are classified into: (i) validation of the model predictions with the available measurements of other investigators, (ii) predicting the influence of each shaped charge parameter on its performance, (iii) comparison between the analytical results with the corresponding results of jetting analysis process of Autodyn-2D hydrocode and (iv) comparison between numerical and analytical results concerning with the influence of each shaped charge factor on its performance.

\section{Validation of the Proposed Model Predictions}

Mohamed [8] has been performed an experimental program using small caliber shaped charges to validate his analytical model results. He also studied the influence of charge type and standoff distance on the performance of the tested shaped charges. The tested charges had an apex angle of $60^{\circ}$, a cone base diameter of 14.9 $\mathrm{mm}$ and a copper liner of thickness $0.8 \mathrm{~mm}$. In addition, He used three types of explosives; these were TNT, RDX and HMX having detonation wave velocities of 6850,8700 and $9110 \mathrm{~m} / \mathrm{s}$, respectively. The tested standoff distances were 15, 30 and $45 \mathrm{~mm}$, respectively. For each tested shaped charge, both the depth of jet penetration into a semi-infinite RHA and the crater radius at target surface were measured.

The present model was fed with the data of each tested shaped charge of Ref. [8]. The model predicts the penetration depth and the crater radius at target surface for each shaped charge. The maximum absolute error between the measured penetration depth of Ref. [8] and the corresponding prediction of the present model is found to be $12.6 \%$ whereas, the maximum absolute error between the measured crater radius at target surface of Ref. [8] and that of the present model is found to be $13.5 \%$. In addition, Figure 5 plots the influence of explosive type on the measured penetration depth of Ref. [8] whereas; Figure 6 plots the influence of explosive type 
Table 5. Input data of monolithic RHA to the hydrocode.

\begin{tabular}{|l|c|l|c|}
\hline \multicolumn{1}{|c|}{ Parameter } & Value & \multicolumn{1}{c|}{ Parameter } & Value \\
\hline Equation of state & linear & Hardening exponent, $\mathrm{n}$ & 0.26 \\
\hline Strength model & Joh.- Cook & Strain rate constant, C & $1.4 \times 10^{-2}$ \\
\hline Reference density $\left(\mathrm{kg} / \mathrm{m}^{3}\right)$ & 7860 & Thermal exponent const., $\mathrm{m}$ & 1.03 \\
\hline Yield strength, A (MPa) & 765 & Reference temperature $(\mathrm{K})$ & 300 \\
\hline Bulk modulus $(\mathrm{GPa})$ & 159 & Shear modulus $(\mathrm{GPa})$ & 82.2 \\
\hline Hardening constant, B (MPa) & 510 & Specific heat $(\mathrm{J} / \mathrm{kg} . \mathrm{K})$ & 477 \\
\hline
\end{tabular}

on the measured crater radius at target surface of the same reference. The corresponding predictions of the proposed model and that of Ref. [8] are plotted on their respective figures. Good agreement is obtained between the predictions of the proposed model and the corresponding measurements of Ref. [8].

Figure 7 plots the influence of the standoff distance on the measured penetration depth of Ref. [8] whereas, Figure 8 plots the influence of the standoff distance on the measured crater radius at target surface. The corresponding predictions of the proposed model and that of Ref. [8] are plotted on their respective figures. The obtained results prove the predictive capabilities of the proposed analytical model.

Another validation of the proposed model was done by comparing the measured penetration capability of the shaped charge model $x x$ into semi-infinite RHA with the prediction of the present model. The proposed model was fed with the geometrical dimensions and material properties of the shaped charge elements. The measured penetration depth by the shaped charge jet into RHA was $69.85 \mathrm{~mm}$ [19] whereas. The predicted penetration depth is 71.1 with an absolute error of $1.78 \%$.

\section{Predicting the Influence of Each Shaped Charge Factor on its Performance}

The proposed model is used to predict the influence of each factor of the shaped charge model $x x$ on its performance. The studied factors are: (i) liner thickness, (ii) liner material, (iii) cone angle, (iv) type of explosive, and (v) stand-off distance between the cone base and target surface. The span for each of the studied factors is listed in Table 1. Figure 9 plots the influence of each factor of shaped charge model $x x$ on the percent of increase or decrease in penetration depth. It is seen from the figure that the factors improving the shaped charge performance are explosive material, cone angle and standoff distance.

The main predictions of the proposed model concerned with the factors affecting the performance of the shaped charge model $x x$ are:

i) The jet penetration depth into RHA increases by $8 \%$ compared with that of the original shaped charge model $x x$ when the wall thickness decreases from $1.2 \mathrm{~mm}$ to $1 \mathrm{~mm}$. The obtained results are similar to that of Saran et al. [4] who deduced experimentally that the decrease in liner thickness had an increasing effect on penetration depth with minor decrease in crater diameter. 
ii) The penetration depth of jet formed from copper liner of the original shaped charge into RHA is greater than that of jet formed from steel liner by $15 \%$. In addition, the predicted crater radius at target surface for copper jet is less that that predicted for steel jet. The predicted results for the penetration depth associated with liner density are similar to that obtained by Cowan et al. [20].

iii) The depth of penetration increases by $11 \%$ compared with that of the original shaped charge model $x x$ when RDX explosive is replaced with HMX type with minor increase in crater diameter. Similar trends to the model predictions are obtained experimentally by Baker et al. [5] who reported that the use of powerful explosive provided more detonation energy and resulted in longer jet and great penetration depth into a target.

iv) The jet penetration depth increases by $27 \%$ compared with that of the original shaped charge when the half of the apex angle decreases from $30^{\circ}$ to $25^{\circ}$ considering no change in the cone radius. The obtained results are similar to that of Green [21] who reported that the formed jet becomes shorter, thicker and less penetrative with the increase of half apex angle.

v) The depth of penetration increases by $16.3 \%$ for the shaped charge with standoff distance of $26 \mathrm{~mm}$ compared with that of the original shaped charge with standoff distance of $19 \mathrm{~mm}$. In addition, the depth of penetration increases with standoff distance when the jet keeps its continuity (not particulated).

\section{Comparing the Analytical Results with that of Jetting Analysis Process}

The mesh sensitivity study for the jetting analysis was performed on the shaped charge model $x x$. It is noticed that the mesh size $0.25 \times 0.35 \mathrm{~mm}$ for explosive charge gave jet mass equal to $2.89 \mathrm{~g}$ when jetting analysis was completed. This result was close to the analytical result which predicted a jet mass of $2.97 \mathrm{~g}$. Therefore, the mesh size of $0.25 \times 0.35 \mathrm{~mm}$ was used for the jetting analysis of $x \mathrm{x}$ shaped charge jet formation.

Mesh sensitivity is also an important issue in jet penetration. The mesh size for griding $\mathrm{RHA}$ has been changed until a penetration depth close to the measured value was predicted. Autodyn-2D hydrocode predicted that the jet penetration into $\mathrm{RHA}$ was $70.4 \mathrm{~mm}$ when the mesh size was $0.25 \times 0.35 \mathrm{~mm}$. This result was close to the measured depth into RHA which was equal to $69.85 \mathrm{~mm}$ [20]. This mesh size for RHA was used when simulating the jet penetration process into Autodyn-2D hydrocode.

Autodyn-2D hydrocode was fed with the data of shaped charge model $x x$. Jetting analysis process is used to predict the parameters associated with jet formation process. Table 6 lists the numerical values of the main parameters associated with jet formation process and the break-up time at which the first jet segmentation occurs. The corresponding predicted results of the proposed model and the maximum absolute errors relative to the analytical results are also listed in the same table. The present table shows that the numerical results obtained by jetting analysis process and the corresponding predictions obtained by the proposed model are in good agreement. In addition, the ratios of jet mass relative to liner mass $\left(\mathrm{mj} / \mathrm{M}_{\mathrm{L}}\right)$ 
Table 6. Comparison between the numerical and analytical results of jet formation process of shaped charge model $x \mathrm{x}$.

\begin{tabular}{|c|l|c|c|c|}
\hline $\begin{array}{c}\text { Ser. } \\
\text { No. }\end{array}$ & \multicolumn{1}{|c|}{ Parameter } & Autodyn-2D & $\begin{array}{c}\text { Proposed } \\
\text { model }\end{array}$ & $\begin{array}{c}\text { Max. } \\
\text { Absolute } \\
\text { error [\%] }\end{array}$ \\
\hline 1 & Jet mass, $\mathrm{m}_{\mathrm{j}}[\mathrm{g}]$ & 2.89 & 2.97 & 2.7 \\
\hline 2 & Liner mass, $\mathrm{M}_{\mathrm{L}}[\mathrm{g}]$ & 15.8 & 16.9 & 6.5 \\
\hline 3 & Ratio of $\left(\mathrm{m}_{\mathrm{j}} / \mathrm{M}_{\mathrm{L}}\right)$ & 18.3 & 17.5 & 4.6 \\
\hline 4 & Max. flow velocity, $\mathrm{V}_{\max }[\mathrm{m} / \mathrm{s}]$ & 2857 & 3098 & 7.8 \\
\hline 5 & Max. stag. velocity, $\mathrm{V}_{1 \max }[\mathrm{m} / \mathrm{s}]$ & 3524 & 3710 & 5.04 \\
\hline 6 & Max. jet velocity, $\mathrm{V}_{\mathrm{jmax}}[\mathrm{m} / \mathrm{s}]$ & 6381 & 6808 & 6.3 \\
\hline 7 & Min. flow velocity, $\mathrm{V}_{\min }[\mathrm{m} / \mathrm{s}]$ & 1957 & 1836 & 6.6 \\
\hline 8 & Min stag. velocity, $\mathrm{V}_{1 \min }[\mathrm{m} / \mathrm{s}]$ & 1689 & 1644 & 2.7 \\
\hline 9 & Min jet velocity, $\mathrm{V}_{\mathrm{jmin}}[\mathrm{m} / \mathrm{s}]$ & 3645 & 3480 & 4.7 \\
\hline 10 & First break-up time, $\mathrm{t}_{\mathrm{b} 1}[\mu \mathrm{s}]$ & 22.6 & 25.8 & 12.4 \\
\hline
\end{tabular}

obtained numerically and analytically are less than $20 \%$ which is similar to that reported by Walter and Zukas [9].

\section{Comparison between Numerical and Analytical Results Concerning with the Influence of each Shaped Charge Factor on its Performance.}

In the following, Table 7 lists the penetration depth into RHA and crater radius at the target surface obtained by Autodyn-2D, the corresponding analytical results of the proposed model and the measured penetration depth of the shaped charge jet into RHA of Ref. [19]. In addition, the absolute error between each of the predicted penetration depths and the measured value is also listed in the same table. The absolute errors between the measured penetration depth and each of that obtained analytically and numerically are 1.79 and $0.93 \%$, respectively.

To predict the influence of each shaped charge factor on its performance, the considered values of each factor listed in Table 1 are fed into hydrocode. The numerical values of penetration depth and crater radius at target surface for each value of the shaped charge factor are listed in Table 8. In addition, the corresponding results obtained by the proposed model are listed in the same table. For each studied factor, good agreement is generally obtained between the results obtained by Autodyn-2D and that of the proposed model; the maximum absolute error is found to be $14.7 \%$ when TNT is used instead of RDX.

It is also seen form the table that the decrease of the half of apex angle and the increase of standoff distance has the greatest effect on the $x x$ shaped charge performance compared with the other shaped charge factors. Figure 10 plots the predicted change of penetration depth obtained by Autodyn-2D and that of the 
Table 7. Comparison between the numerical and analytical results and the measurement of Ref. [19].

\begin{tabular}{|c|c|c|c|c|c|c|}
\hline \multicolumn{3}{|c|}{ Penetration depth, Z [mm] } & \multicolumn{2}{c|}{$\begin{array}{c}\text { Crater radius, } \\
r_{c}[\mathrm{~mm}]\end{array}$} & \multicolumn{2}{c|}{$\begin{array}{c}\text { Abs. Error, } \\
\Delta Z / Z_{\mathrm{m}}[\%]\end{array}$} \\
\hline Model & Autodyn & Exp. [19] & Model & Autodyn & Model & Autodyn \\
\hline 71.1 & 70.4 & 69.85 & 5.46 & 5.46 & 1.79 & 0.93 \\
\hline
\end{tabular}

$Z_{m}$ is the measured penetration depth of $x x$ shaped charge jet into $R H A$.

Table 8. Comparison between the effects of each shaped charge factor on its performance.

\begin{tabular}{|c|c|c|c|c|c|c|}
\hline \multirow{2}{*}{ Parameter } & \multicolumn{2}{|c|}{$\begin{array}{c}\text { Penetration depth, } \\
\mathrm{Z}[\mathrm{mm}]\end{array}$} & \multicolumn{2}{|c|}{$\begin{array}{c}\text { Crater radius, } \\
\mathbf{r}_{\mathrm{C}}[\mathrm{mm}]\end{array}$} & \multirow{2}{*}{$\begin{array}{c}\text { Abs. } \\
\text { Error, } \\
\Delta Z / Z_{P} \text { * } \\
{[\%]}\end{array}$} & \multirow{2}{*}{$\begin{array}{c}\text { Abs. } \\
\text { Error, } \\
\Delta \mathrm{r}_{\mathrm{C}} / \mathrm{r}_{\mathrm{CP}}{ }^{\star \star} \\
{[\%]}\end{array}$} \\
\hline & Autodyn & Model & Autodyn & Model & & \\
\hline \multicolumn{7}{|c|}{ Liner wall thickness } \\
\hline$t_{1}=1.0 \mathrm{~mm}$ & 87.00 & 76.8 & 5 & 5.05 & 13.2 & 1.00 \\
\hline$t_{1}=1.4 \mathrm{~mm}$ & 63.65 & 66.2 & 6.4 & 5.84 & 3.85 & 9.58 \\
\hline \multicolumn{7}{|c|}{ Liner material } \\
\hline Steel & 60.00 & 60.5 & 6.1 & 6.05 & 0.83 & 0.83 \\
\hline \multicolumn{7}{|c|}{ Explosive material } \\
\hline TNT & 36.50 & 42.2 & 5.8 & 5.31 & 14.7 & 9.22 \\
\hline HMX & 68.41 & 78.9 & 6.1 & 5.54 & 13.3 & 10.1 \\
\hline \multicolumn{7}{|c|}{ Half of cone angle (constant radius) } \\
\hline$\alpha=25^{\circ}$ & 85.00 & 90.1 & 5.5 & 5.74 & 5.66 & 4.18 \\
\hline$\alpha=35^{\circ}$ & 59.50 & 56.7 & 6 & 5.18 & 4.93 & 15.8 \\
\hline \multicolumn{7}{|c|}{ Standoff distance } \\
\hline SoD $=26 \mathrm{~mm}$ & 73.50 & 82.7 & 5.5 & 5.06 & 11.1 & 8.69 \\
\hline SoD $=38 \mathrm{~mm}$ & 90.50 & 102.8 & 4.87 & 4.54 & 11.9 & 7.27 \\
\hline
\end{tabular}

* $\left(\boldsymbol{\Delta Z} / \mathbf{Z}_{\mathrm{P}}\right)=\left(\mathrm{Z}_{\mathrm{P}}-\mathrm{Z}_{\mathrm{a}}\right) / \mathrm{Z}_{\mathrm{P}}$; where $\mathrm{Z}_{\mathrm{P}}$ is the predicted penetration depth by the proposed model, and $\mathrm{Z}_{\mathrm{a}}$ is the penetration depth obtained by Autodyn-2D hydrocode.

** $\left(\Delta r_{C} / r_{C P}\right)=\left(r_{C P}-r_{C a}\right) / r_{C P}$; where $r_{C P}$ is the predicted crater radius at target surface by the proposed model, and $\mathrm{r}_{\mathrm{Ca}}$ is the crater radius obtained by Autodyn-2D hydrocode.

proposed model as function of half of apex angle whereas, Fig. 11 plots the predicted crater radius at target surface obtained numerically and analytically with half of apex angle.

Figure 12 compares also between the predicted change of penetration depth obtained by Autodyn-2D and that of the proposed model as function of standoff distance whereas, Fig. 13 plots the change of the predicted crater radii at target surface obtained numerically and analytically with standoff distance. However, the present results in Table 8 prove the predictive capabilities of the proposed model and Autodyn-2D hydrocode, respectively; experimentation is needed to confirm the obtained predicted results. In addition, both research directions of the present work could be used as a quick tool for predicting the influence of shaped charge factors on its performance. 


\section{CONCLUSIONS}

The main conclusions of the present work are summarized as follows:

- The proposed analytical model proves its predictive capability by comparing its predictions with the available experimental measurements of Ref. [8]; the maximum error for the jet penetration depth into RHA is found to be $12.6 \%$.

- The predicted trend of the change of each parameter associated with jet formation process as function of relative axial position of liner is similar to that obtained other investigators [9,12].

- The numerical results obtained by jetting analysis process for jet formation and the corresponding predictions of the proposed model are in good agreement.

- Analysis of the obtained results, analytically and numerically, for the factors affecting the $x x$ shaped charge performance prove that:

o The depth of penetration increases with the decrease of the liner wall thickness.

- Liners of small cone angles achieve deeper penetration into RHA targets at the same standoff distance.

- The increase of the density of liner material results in a deeper penetration into RHA target with a corresponding decrease in crater radius.

- Deeper penetration is attained with a more powerful explosive.

- The depth of penetration increases with standoff distance when the jet keeps its continuity (not particulated).

o The main factors having great influence on the $x x$ shaped charge performance are: apex angle, explosive type and standoff distance.

- Further experiments are needed to confirm the predictive capabilities of the model and Autodyn-2D hydrocode, respectively.

- The research directions of the present work could be used as a quick tool for predicting the influence of shaped charge factors on its performance.

\section{REFERENCES}

[1] P. Y. Chanteret and A. Lichtenberger, "About Varying Shaped Charge Liner Thickness", $17^{\text {th }}$ Int. Symp. on Ballistics, Midrand, South Africa, 23-27 March (1998).

[2] M. Mayseless, E. Hirsch, A. Lindenfield and Me Bar, "Jet Tip and Appendix Dependence on Liner Thickness in $60^{\circ}$ Point Initiated Shaped Charge", $17^{\text {th }}$ Int. Symp. on Ballistics, Midrand, South Africa, 23-27 March (1998).

[3] P.Y. Chanteret, "Theoretical Considerations about Jet Density and Shaped Charge Performance", Proceedings of the $17^{\text {th }}$ Int. Symp. on Ballistics, Midrand, March (1998).

[4] S. Saran, O. Ayisit and M. S. Yavuz, "Experimental Investigations on Aluminum Shaped Charge Liners", Procedia Engineering, Vol. 58, pp. 479-486 (2013).

[5] E. Baker, A. Daniels, B. Fuchs, S. Nicolich, J. Orosz, J. Pham, B. Travers and T. Vuong, "Improved Performance of Shaped Charge Warheads Using More Powerful Explosive Formulations", $17^{\text {th }}$ Int. Symp. on Ballistics, Midrand, South Africa, 23-27 March (1998). 
[6] M. Partanian and R.N. Pralhad, "Estimation of Temperature and other Flow Parameters Distribution within the Hollow Charge (HEAT) Projectile', Int. J. Appl. Mathematics, Vol. 24, No. 3, pp. 305-322 (2011).

[7] C. Chuang, W. Xiaoming, L. Wenbin and L. Weibing, "Research on Jet Penetration Law of Biconical Liner Shaped Charge Under Large Standoff Distance Condition", $27^{\text {th }}$ Int. Symp. on Ballistics, Freiburg, Germany, April 2226 (2013).

[8] S.Y. Mohamed, "Factors Affecting the Shaped Charge Performance", M. Sc. Thesis, M.T.C., Cairo, Egypt (2000).

[9] P. Walters and J. A. Zukas, "Fundamentals of Shaped Charges", Wiley Interscience Publication, John Wiley and Sons, New York, U.S.A. (1989).

[10] E. Hennequin, "Modeling of Shaped Charge Jet Break-up", Propellants, Explosives, Pyrotechnics Vol. 21, pp. 181-185 (1996).

[11] S.A. Ahmed, " Evaluation of Shaped Charge Performance under Variable Liner Parameters", M. Sc. Thesis, M.T.C., Cairo, Egypt, to be discussed in (2014).

[12] L. Gui-Xi, "The Simplified Model for Predicting Shaped Charge Jet Parameters", Propellants, Explosives, Pyrotechnics, Vol. 20, pp. 279-282 (1995).

[13] P. Y. Chanteret, ,"Considerations About the Analytical Modeling of Shaped Charges", Propellants, Explosives, Pyrotechnics, Vol. 18, pp. 337-344 (1993).

[14] Anon, "Rocket Warheads and Fuzes", Military Technical College Printed lectures Nos. 785 and 786, M.T.C., Cairo, Egypt (1962).

[15] W. Walters, W.S. Flis and P.C. Chou, "A Survey of Shaped-Charge Jet Penetration Models", Int. J. Impact Engng., Vol. 7, No 3, pp. 307-325 (1988).

[16] J. Petit, V. Jeanclaude and C. Fressengeas, "Breakup of Copper shapedcharge jets: Experiment, numerical simulations and analytical modeling", J. Appl. Physics, Vol. 98, pp. 123521-1: 123521-10 (2005).

[17] Specification book, research for developing Shaped charge model Xx, Sakr factory (2000).

[18] Autodyn-2D, 'Theory Manual', Revision 3.0, Century Dynamics, U.S.A. (1997).

[19] TM 43-0001-28, 'Grenade: General Purpose xx', Technical Manual of Army Ammunition Data Sheets', pp. 8-51, April (1994).

[20] K.G. Cowan, K.J. Mawella, D.J. Standing, B. Bourne, J. Jones, and A. Kitney, "Analytical Code and Hydrocode Modelling and Experimental Characterisation of Shaped Charges Containing Tungsten Liners", 17th Int. Symp. on Ballistics, Midrand, South Africa, 23-27 March (1998).

[21] P.R. Green, 'Ammunition for the Land Battle', UK: BRASSEY'S, 4th Edition. Vol. 4. (1990). 


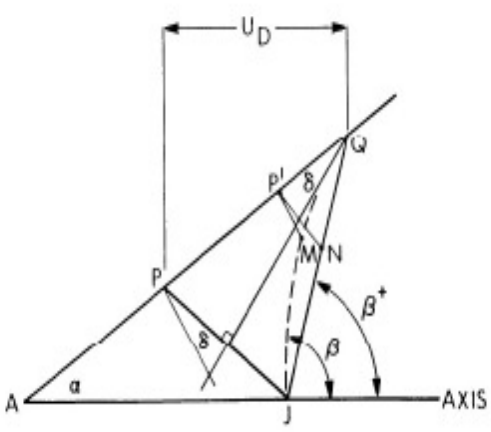

Fig. 1. The collapse process of a variable velocity liner [9].

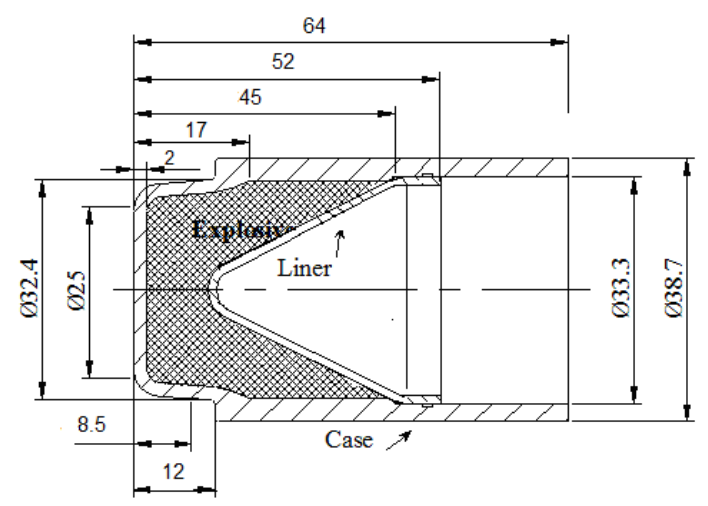

Fig. 3. The conical shaped charge Model xx [17].

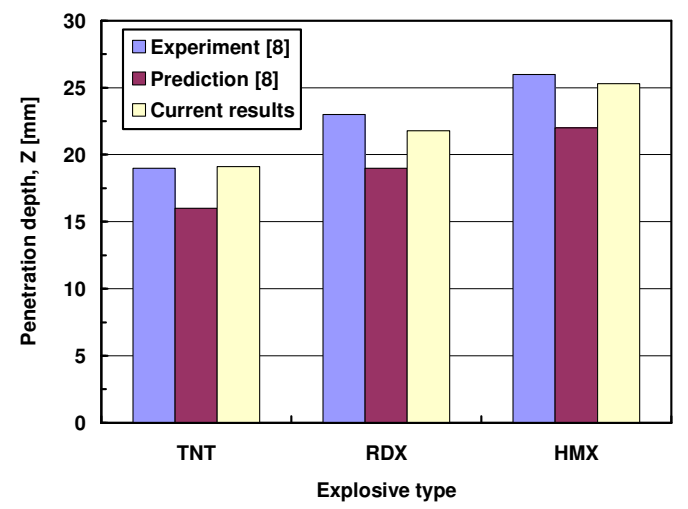

Fig. 5. Comparison between the predicted and measured penetration depths of Ref. [8] and the model predictions as function of explosive type.
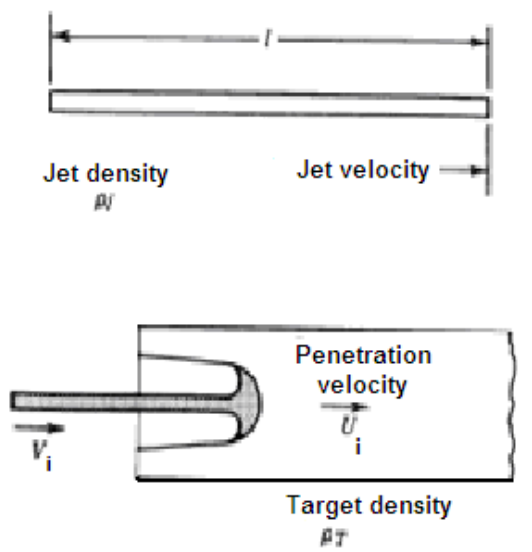

Fig. 2. Jet penetration into target [9].

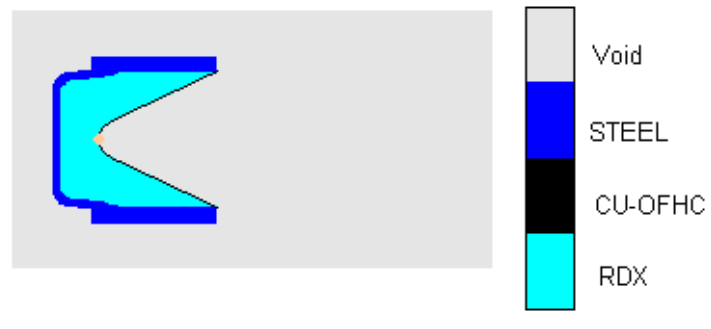

Fig. 4. Simulating of the jetting analysis process in Autodyn-2D code.

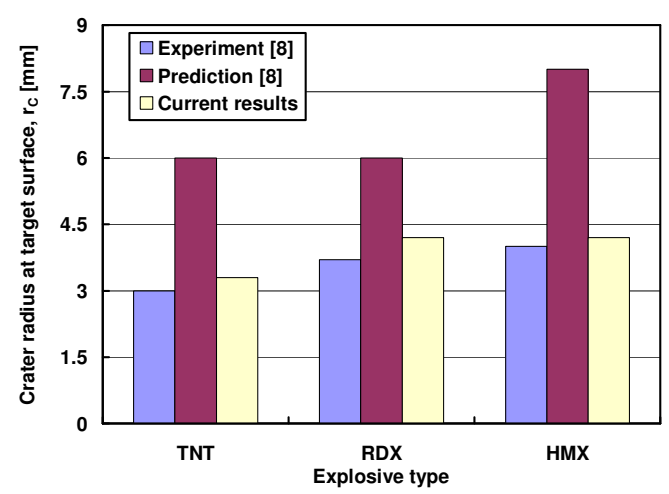

Fig. 6. Comparison between the predicted and measured crater radii of Ref. [8] and the model predictions as function of expl. type. 


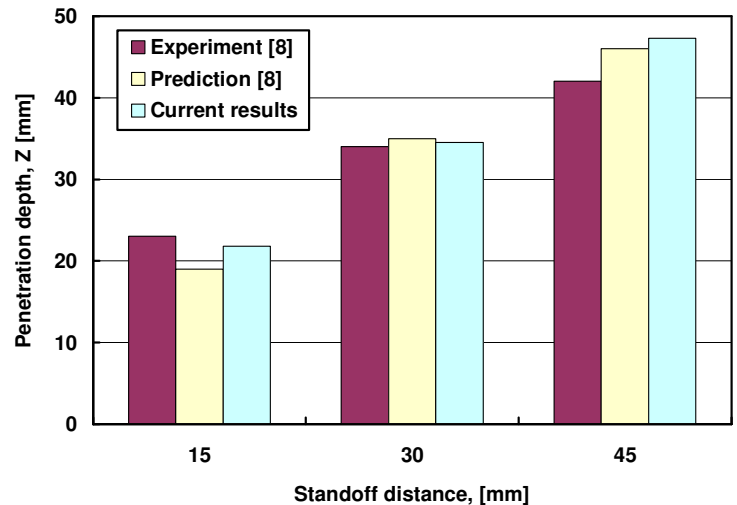

Fig. 7. Comparison between the predicted and measured penetration depths of Ref. [8] and the model predictions as function of standoff distance.

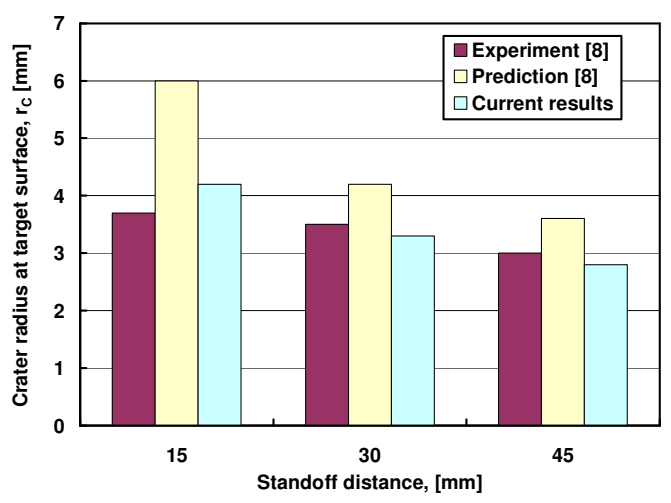

Fig.8. Comparison between the predicted and measured crater radii of Ref. [8] and the model predictions as function of standoff distance.

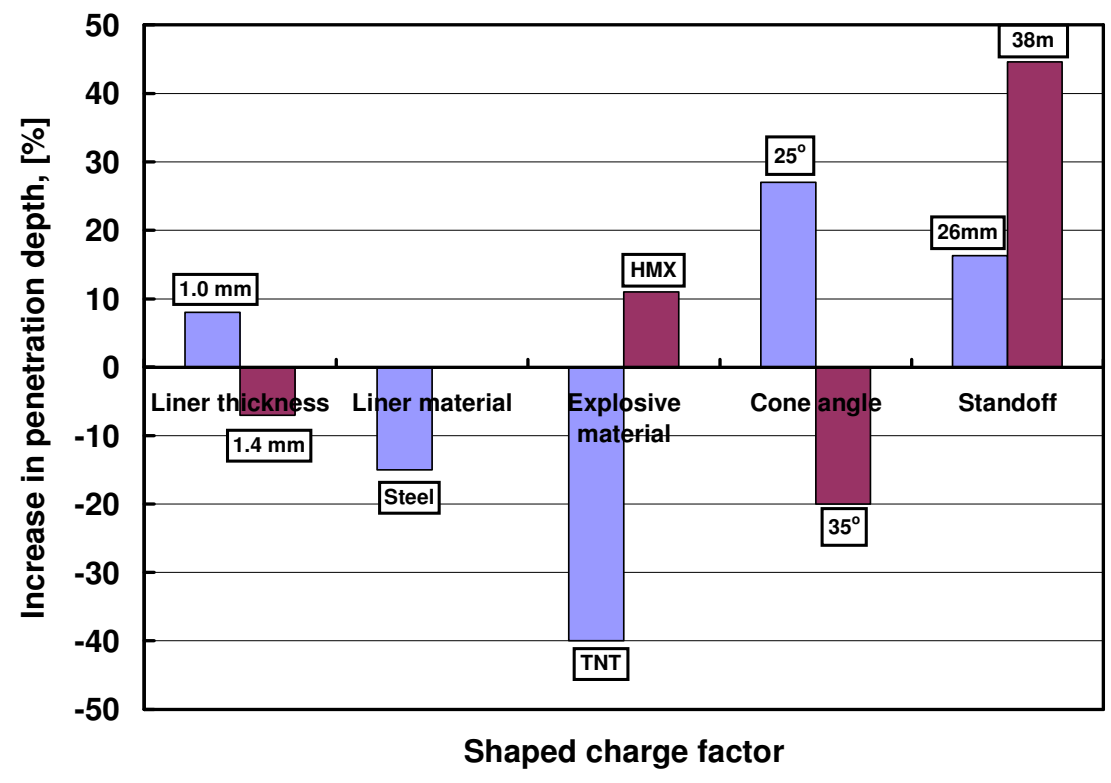

Fig. 9. The predicted influence of each factor of shaped charge model $x x$ on its performance.

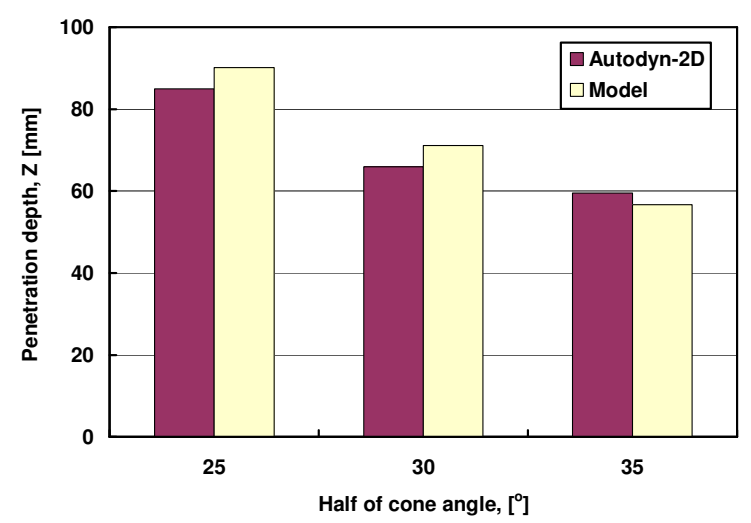

Fig. 10. Comparison between the penetration depth as function of half cone angle obtained by Autodyn-2D and model.

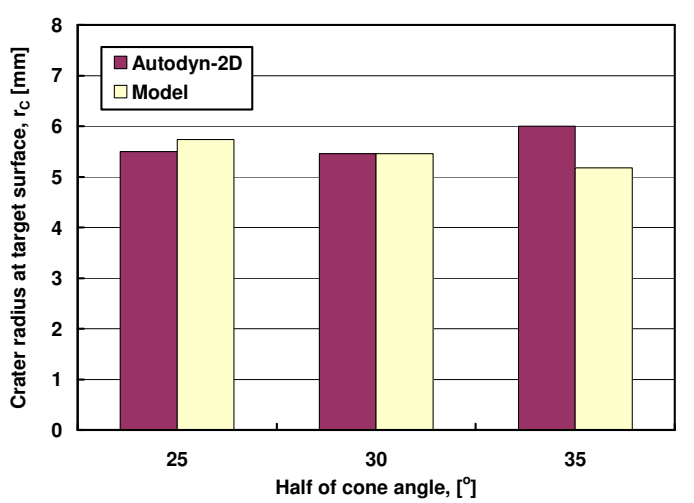

Fig. 11. Comparison between the crater radius as function of half cone angle obtained by Autodyn-2D and model. 


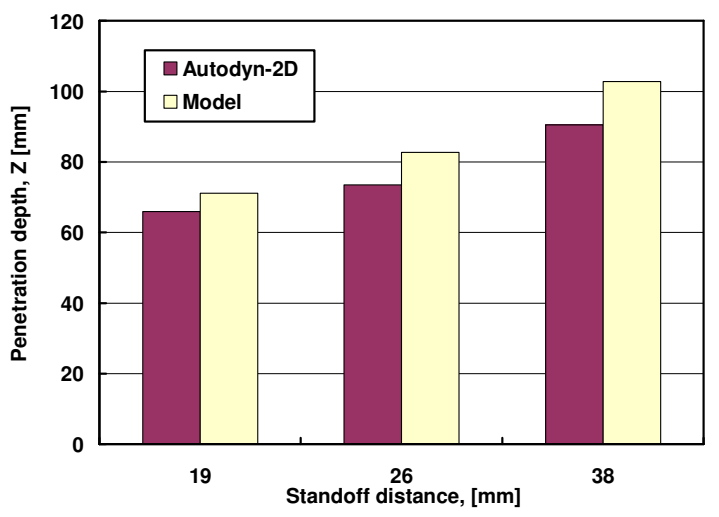

Fig. 12. Comparison between the penet. depth as function of standoff obtained by Autodyn$2 \mathrm{D}$ and the proposed model.

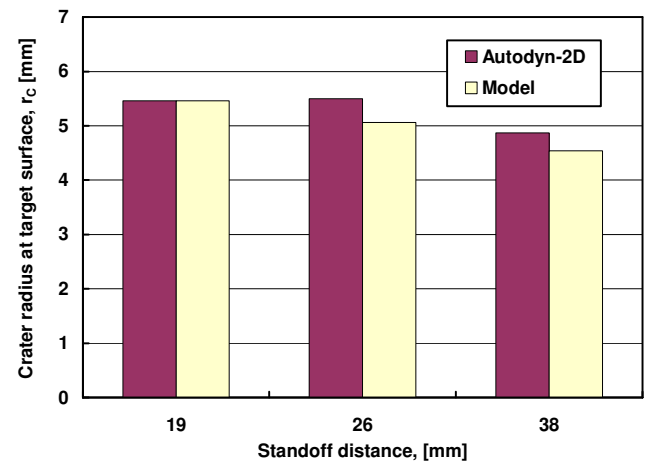

Fig. 13. Comparison between the crater radius as function of standoff obtained by Autodyn- 2D and the proposed model. 